Supporting Information for

\title{
The Significance of Chemical Environment of an Element in Non-Adiabatic Molecular Dynamics: Feature Selection and Dimensionality Reduction with Machine Learning
}

Wei Bin How, ${ }^{1}$ Bipeng Wang, ${ }^{2}$ Weibin Chu, ${ }^{3}$ Alexandre Tkatchenko, ${ }^{4}$ Oleg V. Prezhdo ${ }^{2,3,5, *}$

${ }^{1}$ Division of Chemistry and Biological Chemistry, School of Physical and Mathematical Sciences, Nanyang Technological University, 637371, Singapore

${ }^{2}$ Department of Chemical Engineering, University of Southern California, Los Angeles, CA 90089, USA

${ }^{3}$ Department of Chemistry, University of Southern California, Los Angeles, CA 90089, USA

${ }^{4}$ Department of Physics and Materials Science, University of Luxembourg, L-1511 Luxembourg City, Luxembourg

${ }^{5}$ Department of Physics and Astronomy, University of Southern California, Los Angeles, CA 90089, USA

\section{Simulation Details}

The ab initio DFT calculations were performed on a pristine $\mathrm{CsPbI}_{3}$ system, with the tetragonal phase represented by a $(\sqrt{2} \times \sqrt{2} \times 2)$ unit cell with the lattice constants $9.02 \AA \times 9.02 \AA \times 12.76$ $\AA$. The calculation of the target variables, such as bandgap and nonadiabatic coupling (NAC), geometry optimization and generation of the trajectory dataset was performed using the Vienna $\mathrm{ab}$ initio Simulation Package (VASP) and the Perdew-Burke-Ernzerhof (PBE) exchangecorrelation functional. ${ }^{1,2}$ For the PBE exchange-correlation functional, the projected-augmented wave method is used for interactions between electrons and ion cores. The NACs were calculated from the overlap between two wavefunctions at adjacent timesteps using the CA-NAC package. ${ }^{3,4}$ The expression for the calculation of the NAC between two wave functions is as follows:

$$
d_{j i}=-i \hbar\left\langle\varphi_{j}(\boldsymbol{r}, \boldsymbol{R}(t))\left|\nabla_{\boldsymbol{R}}\right| \varphi_{i}(\boldsymbol{r}, \boldsymbol{R}(t))\right\rangle \frac{\mathrm{d} \boldsymbol{R}}{\mathrm{dt}}
$$

\footnotetext{
${ }^{*}$ Corresponding Author. Email: prezhdo@usc.edu
} 


$$
\begin{gathered}
=-i \hbar \frac{\left\langle\varphi_{j}(\boldsymbol{r}, \boldsymbol{R}(t))\left|\nabla_{\boldsymbol{R}} H(\mathbf{R}(t))\right| \varphi_{i}(\boldsymbol{r}, \boldsymbol{R}(t))\right\rangle}{E_{i}-E_{j}} \frac{d \boldsymbol{R}}{\mathrm{dt}} \\
=-i \hbar\left\langle\varphi_{j}(\boldsymbol{r}, \boldsymbol{R}(t))\left|\frac{\partial}{\partial t}\right| \varphi_{i}(\boldsymbol{r}, \boldsymbol{R}(t))\right\rangle \\
\approx-\frac{i \hbar}{2 \Delta t}\left\{\left\langle\varphi_{j}(\boldsymbol{r}, \boldsymbol{R}(t)) \mid \varphi_{i}(\boldsymbol{r}, \boldsymbol{R}(t+\Delta t))\right\rangle-\left\langle\varphi_{j}(\boldsymbol{r}, \boldsymbol{R}(t+\Delta t)) \mid \varphi_{i}(\boldsymbol{r}, \boldsymbol{R}(t))\right\rangle\right\}
\end{gathered}
$$

Although the above expression is written in terms of Kohn-Sham (KS) orbitals, the calculations for NACs are performed using the many-particle Slater determinant basis, with the NAC between Slater determinants being reduced to the NAC between KS orbitals.

Geometry relaxation was first performed at $0 \mathrm{~K}$, followed by heating to $300 \mathrm{~K}$. After reaching thermal equilibrium, a $7000 \mathrm{fs}$ trajectory was generated in a microcanonical ensemble with a $1 \mathrm{fs}$ timestep. The decoherence-induced surface hopping (DISH) method was used to simulate the nonradiative charge recombination process. ${ }^{5}$ The pure-dephasing times used in DISH were obtained via second order cumulant approximation of the optical response theory. ${ }^{6,7}$ The nonadiabatic molecular dynamics (NA-MD) simulations were carried out using the PYXAID software. ${ }^{8,9}$ Classical path approximation was used, as it allows for the computation of the NAMD at a significantly reduced computational load by substituting multiple excited state MD trajectories with a single ground state trajectory. This approximation is valid in situations whereby MD is driven by thermal fluctuations and when the difference between ground and excited state geometries are insignificant compared to the fluctuation amplitude.

\section{Kernel Ridge Regressor}

Kernel ridge regression is an extension of ridge regression, which involves introduction of a L2 regularization term, $\|w\|^{2}$, whereby $w$ represents the weights of the model, to the ordinary least squares cost function of a simple linear regression model such that the total cost function is as follow:

$$
C=\frac{1}{2} \sum_{i}\left(y_{i}-w^{T} x_{i}\right)^{2}+\frac{1}{2} \lambda\|w\|^{2}
$$

$\lambda$ represents the regularization factor. The introduction of the regularization term is to decrease overfitting, by penalizing a complex model by shrinking the value of the weights. 
For the case of kernel ridge regression, the data are replaced by their feature vectors: $x_{i} \rightarrow \Phi_{i}=$ $\Phi\left(x_{i}\right)$, induced by a kernel, whereby $k\left(x_{i}, x_{j}\right)=\Phi\left(x_{i}\right)^{T} \Phi\left(x_{j}\right)$. This way, it allows for the representation of the original data in higher dimensions, perhaps much higher than the total number of observations in the dataset. Despite the high dimensionality, the individual features need not be accessed directly, allowing for a reduction in computational load, thanks to the kernel trick, whereby the prediction of a new observation, $x$, can be computed without access to the feature vectors by projecting it onto the solution of $w$.

Kernel Trick: $\quad\left(P^{-1}+B^{T} R^{-1} B\right)^{-1} B^{T} R^{-1}=P B^{T}\left(B P B^{T}+R\right)^{-1}$

Prediction of $\mathrm{x}: \quad \quad \quad w=\left(\lambda I_{d}+\Phi \Phi^{T}\right)^{-1} \Phi y=\Phi\left(\Phi^{T} \Phi+\lambda I_{n}\right)^{-1} y$

$$
y=w^{T} \Phi=\mathrm{y}\left(\Phi^{T} \Phi+\lambda I_{n}\right)^{-1} \Phi^{T} \Phi(x)=y\left(K+\lambda I_{n}\right)^{-1} \kappa(x)
$$

where $K\left(b x_{i}, b x_{j}\right)=\Phi\left(x_{i}\right)^{T} \Phi\left(x_{j}\right)$ and $\kappa(x)=K\left(x_{i}, x_{j}\right)$.

In this study, the Laplacian kernel was used, $K(x, y)=e^{-\gamma\|x-y\|_{1}}$, whereby $\mathrm{x}$ and $\mathrm{y}$ represented two separate input vectors, and $\|x-y\|_{1}$ represents the Manhattan distance between them.

The kernel ridge regressor can be optimized analytically, without the need for gradient descent, which might lead to inconsistent training outcomes due to the presence of local and global minima. 


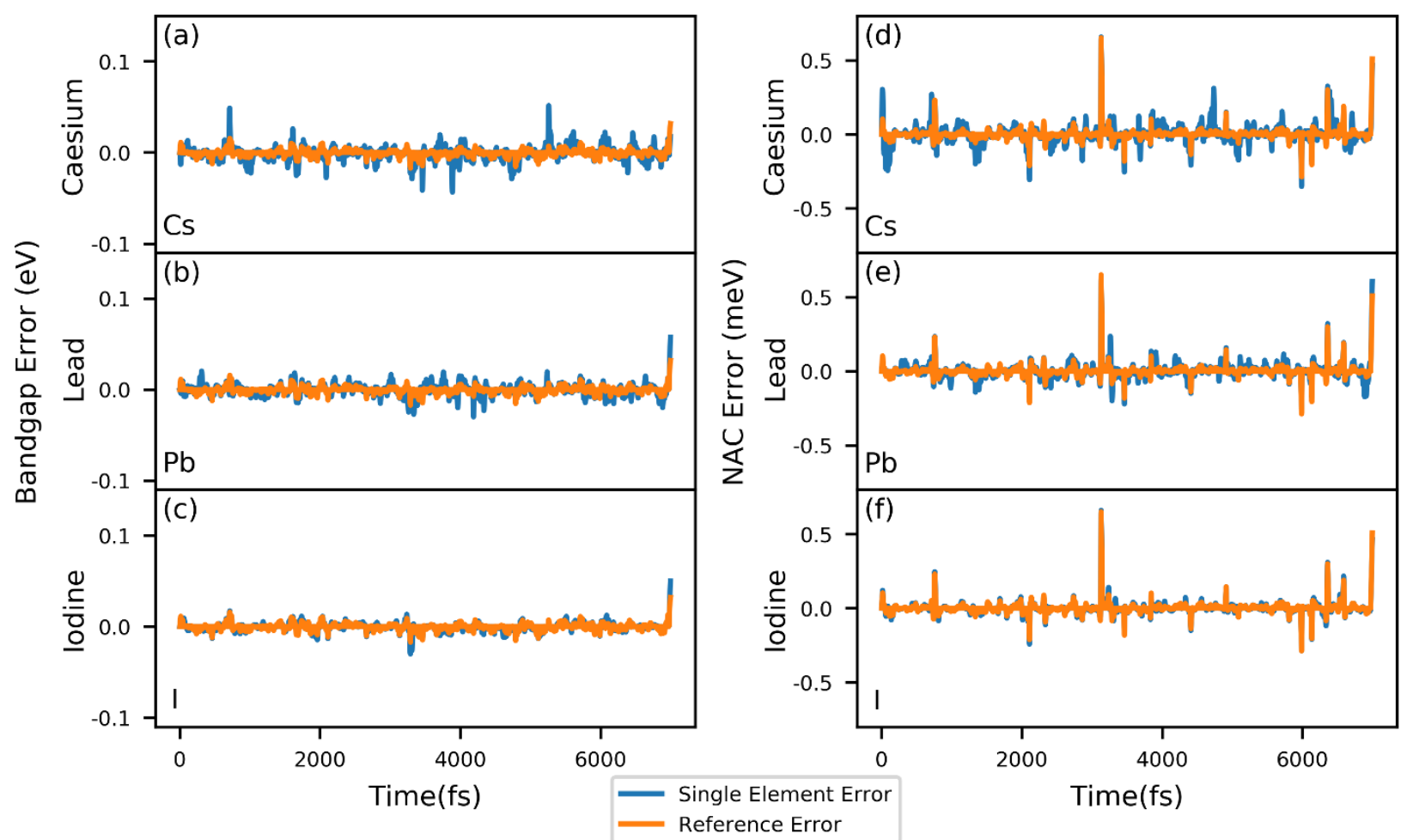

Figure S1: Individual element KRR model errors plotted against the reference error, which is the error made by the model trained on the features of all three elements. (a, b, c) refer to models trained using only caesium, lead or iodine features, respectively, for the prediction of bandgap. (d, e, f) refer to models trained using only caesium, lead or iodine features, respectively, for the prediction of non-adiabatic coupling (NAC). 


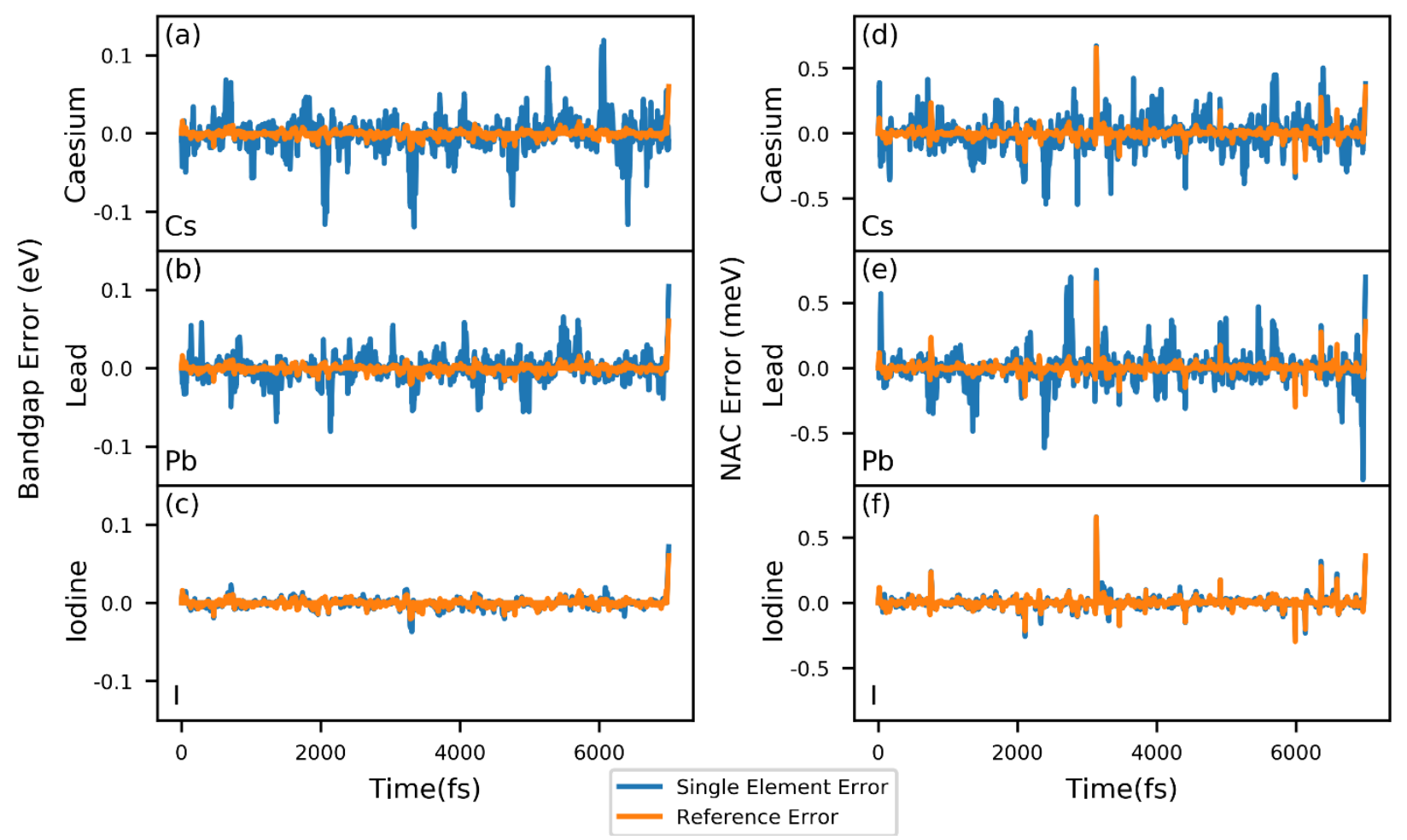

Figure S2: Error of KRR models built based on only one optimal feature of one given element, plotted against the reference error, which is the error made by a model trained on the optimal features of all three elements, Table 2. The optimal feature is selected to have the highest mutual information with the corresponding property, i.e. either bandgap of non-adiabatic coupling (NAC). The feature is defined by the $\theta_{\mathrm{s}}$ and $\mathrm{R}_{\mathrm{s}}$ values, Eq. (1). (a, b, c) refer to models trained using only caesium, lead or iodine optimal features, respectively, for the prediction of bandgap. (d, e, f) refer to models trained using only caesium, lead or iodine optimal features, respectively, for the prediction of NAC. 
Table S1: Full table of the mean mutual information (MI) between band gap and the features of caesium with different $\theta_{s}$ and $R_{s}$ values, Eq. (1).

\begin{tabular}{|l|l|l|}
\hline $\boldsymbol{\theta} s$ & $\mathbf{R}_{\mathbf{s}}$ & MI with Bandgap \\
\hline $\mathrm{Pb}-\mathrm{Cs}-\mathrm{I}$ & Cs-I & 1.552 \\
\hline $\mathrm{Pb}-\mathrm{Cs}-\mathrm{Pb}$ & Cs-I & 1.551 \\
\hline Cs-Cs-I & Cs-I & 1.550 \\
\hline Cs-Cs-Cs & Cs-I & 1.550 \\
\hline Cs-Cs-Pb & Cs-I & 1.549 \\
\hline I-Cs-I & Cs-Cs & 1.540 \\
\hline I-Cs-I & Cs-I & 1.532 \\
\hline I-Cs-I & Cs-Pb & 1.529 \\
\hline Pb-Cs-Pb & Cs-Pb & 1.527 \\
\hline Pb-Cs-I & Cs-Pb & 1.518 \\
\hline Cs-Cs-Cs & Cs-Pb & 1.516 \\
\hline Cs-Cs-I & Cs-Pb & 1.516 \\
\hline Cs-Cs-Pb & Cs-Pb & 1.503 \\
\hline Pb-Cs-Pb & Cs-Cs & 1.493 \\
\hline Pb-Cs-I & Cs-Cs & 1.481 \\
\hline Cs-Cs-Cs & Cs-Cs & 1.480 \\
\hline Cs-Cs-I & Cs-Cs & 1.480 \\
\hline Cs-Cs-Pb & Cs-Cs & 1.454 \\
\hline
\end{tabular}

Table S2: Full table of the mean mutual information (MI) between non-adiabatic coupling (NAC) and the features of caesium with different $\theta_{s}$ and $R_{s}$ values, Eq. (1).

\begin{tabular}{|l|l|l|}
\hline $\boldsymbol{\theta}_{\mathbf{s}}$ & $\mathbf{R}_{\mathbf{s}}$ & MI with NAC \\
\hline Cs-Cs-Cs & Cs-I & 1.485 \\
\hline Cs-Cs-I & Cs-I & 1.485 \\
\hline Pb-Cs-I & Cs-I & 1.485 \\
\hline Pb-Cs-Pb & Cs-I & 1.475 \\
\hline Cs-Cs-Cs & Cs-I & 1.465 \\
\hline I-Cs-I & Cs-I & 1.465 \\
\hline I-Cs-I & Cs-Cs & 1.463 \\
\hline Cs-Cs-I & Cs-Pb & 1.461 \\
\hline Cs-Cs-Cs & Cs-Pb & 1.460 \\
\hline Pb-Cs-Pb & Cs-Pb & 1.459 \\
\hline I-Cs-I & Cs-Pb & 1.456 \\
\hline Pb-Cs-I & Cs-Pb & 1.455 \\
\hline
\end{tabular}




\begin{tabular}{|l|l|l|}
\hline Cs-Cs-Pb & Cs-Pb & 1.453 \\
\hline Cs-Cs-I & Cs-Cs & 1.438 \\
\hline Cs-Cs-Cs & Cs-Cs & 1.438 \\
\hline Cs-Cs-Pb & Cs-Cs & 1.432 \\
\hline Pb-Cs-I & Cs-Cs & 1.425 \\
\hline $\mathrm{Pb}-\mathrm{Cs}-\mathrm{Pb}$ & Cs-Cs & 1.421 \\
\hline
\end{tabular}

Table S3: Full table of the mean mutual information (MI) between bandgap and the features of lead with different $\theta_{\mathrm{s}}$ and $\mathrm{R}_{\mathrm{s}}$ values, Eq. (1).

\begin{tabular}{|l|l|l|}
\hline $\boldsymbol{\theta}_{\text {s }}$ & $\mathbf{R}_{\mathbf{s}}$ & MI with Bandgap \\
\hline $\mathrm{I}-\mathrm{Pb}-\mathrm{I}$ & $\mathrm{Pb}-\mathrm{Cs}$ & 1.614 \\
\hline $\mathrm{Cs}-\mathrm{Pb}-\mathrm{Pb}$ & $\mathrm{Pb}-\mathrm{Pb}$ & 1.607 \\
\hline $\mathrm{Cs}-\mathrm{Pb}-\mathrm{Pb}$ & $\mathrm{Pb}-\mathrm{Cs}$ & 1.591 \\
\hline $\mathrm{Cs}-\mathrm{Pb}-\mathrm{Cs}$ & $\mathrm{Pb}-\mathrm{Pb}$ & 1.589 \\
\hline $\mathrm{Pb}-\mathrm{Pb}-\mathrm{I}$ & $\mathrm{Pb}-\mathrm{Pb}$ & 1.586 \\
\hline $\mathrm{Pb}-\mathrm{Pb}-\mathrm{Pb}$ & $\mathrm{Pb}-\mathrm{Pb}$ & 1.586 \\
\hline $\mathrm{Pb}-\mathrm{Pb}-\mathrm{Pb}$ & $\mathrm{Pb}-\mathrm{Cs}$ & 1.585 \\
\hline $\mathrm{Cs}-\mathrm{Pb}-\mathrm{Cs}$ & $\mathrm{Pb}-\mathrm{Cs}$ & 1.581 \\
\hline $\mathrm{Pb}-\mathrm{Pb}-\mathrm{I}$ & $\mathrm{Pb}-\mathrm{Cs}$ & 1.581 \\
\hline $\mathrm{Cs}-\mathrm{Pb}-\mathrm{I}$ & $\mathrm{Pb}-\mathrm{Pb}$ & 1.579 \\
\hline $\mathrm{Cs}-\mathrm{Pb}-\mathrm{I}$ & $\mathrm{Pb}-\mathrm{Cs}$ & 1.577 \\
\hline $\mathrm{I}-\mathrm{Pb}-\mathrm{I}$ & $\mathrm{Pb}-\mathrm{Pb}$ & 1.566 \\
\hline $\mathrm{Cs}-\mathrm{Pb}-\mathrm{Pb}$ & $\mathrm{Pb}-\mathrm{I}$ & 1.531 \\
\hline $\mathrm{Cs}-\mathrm{Pb}-\mathrm{Cs}$ & $\mathrm{Pb}-\mathrm{I}$ & 1.527 \\
\hline $\mathrm{Pb}-\mathrm{Pb}-\mathrm{I}$ & $\mathrm{Pb}-\mathrm{I}$ & 1.522 \\
\hline $\mathrm{Pb}-\mathrm{Pb}-\mathrm{Pb}$ & $\mathrm{Pb}-\mathrm{I}$ & 1.516 \\
\hline $\mathrm{Cs}-\mathrm{Pb}-\mathrm{I}$ & $\mathrm{Pb}-\mathrm{I}$ & 1.510 \\
\hline $\mathrm{I}-\mathrm{Pb}-\mathrm{I}$ & $\mathrm{Pb}-\mathrm{I}$ & 1.510 \\
\hline
\end{tabular}

Table S4: Full table of the mean mutual information (MI) between non-adiabatic coupling (NAC) and the features of lead with different $\theta_{\mathrm{s}}$ and $\mathrm{R}_{\mathrm{s}}$ values, Eq. (1).

\begin{tabular}{|l|l|l|}
\hline $\boldsymbol{\theta}_{\mathbf{s}}$ & $\mathbf{R}_{\mathbf{s}}$ & MI with NAC \\
\hline $\mathrm{Pb}-\mathrm{Pb}-\mathrm{Pb}$ & $\mathrm{Pb}-\mathrm{Cs}$ & 1.536 \\
\hline $\mathrm{Pb}-\mathrm{Pb}-\mathrm{I}$ & $\mathrm{Pb}-\mathrm{Cs}$ & 1.535 \\
\hline $\mathrm{Cs}-\mathrm{Pb}-\mathrm{I}$ & $\mathrm{Pb}-\mathrm{Pb}$ & 1.533 \\
\hline $\mathrm{Cs}-\mathrm{Pb}-\mathrm{Cs}$ & $\mathrm{Pb}-\mathrm{Cs}$ & 1.533 \\
\hline
\end{tabular}




\begin{tabular}{|l|l|l|}
\hline I-Pb-I & Pb-Cs & 1.529 \\
\hline $\mathrm{Cs}-\mathrm{Pb}-\mathrm{I}$ & $\mathrm{Pb}-\mathrm{Cs}$ & 1.521 \\
\hline $\mathrm{Cs}-\mathrm{Pb}-\mathrm{Pb}$ & $\mathrm{Pb}-\mathrm{Cs}$ & 1.519 \\
\hline $\mathrm{Cs}-\mathrm{Pb}-\mathrm{Pb}$ & $\mathrm{Pb}-\mathrm{Pb}$ & 1.517 \\
\hline $\mathrm{Pb}-\mathrm{Pb}-\mathrm{Pb}$ & $\mathrm{Pb}-\mathrm{Pb}$ & 1.516 \\
\hline $\mathrm{Cs}-\mathrm{Pb}-\mathrm{Cs}$ & $\mathrm{Pb}-\mathrm{Pb}$ & 1.513 \\
\hline $\mathrm{Pb}-\mathrm{Pb}-\mathrm{I}$ & $\mathrm{Pb}-\mathrm{Pb}$ & 1.512 \\
\hline $\mathrm{I}-\mathrm{Pb}-\mathrm{I}$ & $\mathrm{Pb}-\mathrm{Pb}$ & 1.509 \\
\hline $\mathrm{I}-\mathrm{Pb}-\mathrm{I}$ & $\mathrm{Pb}-\mathrm{I}$ & 1.479 \\
\hline $\mathrm{Pb}-\mathrm{Pb}-\mathrm{Pb}$ & $\mathrm{Pb}-\mathrm{I}$ & 1.478 \\
\hline $\mathrm{Cs}-\mathrm{Pb}-\mathrm{I}$ & $\mathrm{Pb}-\mathrm{I}$ & 1.476 \\
\hline $\mathrm{Cs}-\mathrm{Pb}-\mathrm{Pb}$ & $\mathrm{Pb}-\mathrm{I}$ & 1.471 \\
\hline $\mathrm{Pb}-\mathrm{Pb}-\mathrm{I}$ & $\mathrm{Pb}-\mathrm{I}$ & 1.465 \\
\hline $\mathrm{Cs}-\mathrm{Pb}-\mathrm{Cs}$ & $\mathrm{Pb}-\mathrm{I}$ & 1.461 \\
\hline
\end{tabular}

Table S5: Full table of the mean mutual information (MI) between bandgap and the features of iodine with different $\theta_{\mathrm{s}}$ and $\mathrm{R}_{\mathrm{s}}$ values, Eq. (1).

\begin{tabular}{|l|l|l|}
\hline $\boldsymbol{\theta}_{\text {s }}$ & $\mathbf{R}_{\mathbf{s}}$ & MI with Bandgap \\
\hline $\mathrm{Pb}-\mathrm{I}-\mathrm{I}$ & $\mathrm{I}-\mathrm{Cs}$ & 1.589 \\
\hline $\mathrm{I}-\mathrm{I}-\mathrm{I}$ & $\mathrm{I}-\mathrm{I}$ & 1.586 \\
\hline $\mathrm{Pb}-\mathrm{I}-\mathrm{Pb}$ & $\mathrm{I}-\mathrm{I}$ & 1.585 \\
\hline $\mathrm{Pb}-\mathrm{I}-\mathrm{I}$ & $\mathrm{I}-\mathrm{I}$ & 1.585 \\
\hline $\mathrm{Cs}-\mathrm{I}-\mathrm{Cs}$ & $\mathrm{I}-\mathrm{I}$ & 1.584 \\
\hline $\mathrm{Cs}-\mathrm{I}-\mathrm{Pb}$ & $\mathrm{I}-\mathrm{Cs}$ & 1.582 \\
\hline $\mathrm{Cs}-\mathrm{I}-\mathrm{Pb}$ & $\mathrm{I}-\mathrm{I}$ & 1.580 \\
\hline $\mathrm{Cs}-\mathrm{I}-\mathrm{I}$ & $\mathrm{I}-\mathrm{I}$ & 1.579 \\
\hline $\mathrm{C}-\mathrm{I}-\mathrm{I}$ & $\mathrm{I}-\mathrm{Cs}$ & 1.574 \\
\hline $\mathrm{Cs}-\mathrm{I}-\mathrm{I}$ & $\mathrm{I}-\mathrm{Pb}$ & 1.568 \\
\hline $\mathrm{I}-\mathrm{I}-\mathrm{I}$ & $\mathrm{I}-\mathrm{Cs}$ & 1.568 \\
\hline $\mathrm{Pb}-\mathrm{I}-\mathrm{Pb}$ & $\mathrm{I}-\mathrm{Cs}$ & 1.566 \\
\hline $\mathrm{Pb}-\mathrm{I}-\mathrm{I}$ & $\mathrm{I}-\mathrm{Pb}$ & 1.566 \\
\hline $\mathrm{Cs}-\mathrm{I}-\mathrm{Cs}$ & $\mathrm{I}-\mathrm{Pb}$ & 1.565 \\
\hline $\mathrm{Cs}-\mathrm{I}-\mathrm{Pb}$ & $\mathrm{I}-\mathrm{Pb}$ & 1.562 \\
\hline $\mathrm{Cs}-\mathrm{I}-\mathrm{Cs}$ & $\mathrm{I}-\mathrm{Cs}$ & 1.562 \\
\hline $\mathrm{Pb}-\mathrm{I}-\mathrm{Pb}$ & $\mathrm{I}-\mathrm{Pb}$ & 1.561 \\
\hline $\mathrm{I}-\mathrm{I}-\mathrm{I}$ & $\mathrm{I}-\mathrm{Pb}$ & 1.559 \\
\hline
\end{tabular}


Table S6: Full table of the mean mutual information (MI) between non-adiabatic coupling (NAC) and the features of iodine with different $\theta_{\mathrm{s}}$ and $\mathrm{R}_{\mathrm{s}}$ values, Eq. (1).

\begin{tabular}{|l|l|l|}
\hline $\boldsymbol{\theta}_{\text {s }}$ & $\mathbf{R}_{\mathbf{s}}$ & MI with NAC \\
\hline $\mathrm{I}-\mathrm{I}-\mathrm{I}$ & $\mathrm{I}-\mathrm{Cs}$ & 1.552 \\
\hline $\mathrm{Pb}-\mathrm{I}-\mathrm{Pb}$ & $\mathrm{I}-\mathrm{I}$ & 1.551 \\
\hline $\mathrm{Pb}-\mathrm{I}-\mathrm{Pb}$ & $\mathrm{I}-\mathrm{Cs}$ & 1.549 \\
\hline $\mathrm{Cs}-\mathrm{I}-\mathrm{Cs}$ & $\mathrm{I}-\mathrm{Pb}$ & 1.549 \\
\hline $\mathrm{I}-\mathrm{I}-\mathrm{I}$ & $\mathrm{I}-\mathrm{I}$ & 1.547 \\
\hline $\mathrm{Cs}-\mathrm{I}-\mathrm{Cs}$ & $\mathrm{I}-\mathrm{I}$ & 1.546 \\
\hline $\mathrm{Cs}-\mathrm{I}-\mathrm{I}$ & $\mathrm{I}-\mathrm{Cs}$ & 1.543 \\
\hline $\mathrm{Cs}-\mathrm{I}-\mathrm{Cs}$ & $\mathrm{I}-\mathrm{Pb}$ & 1.539 \\
\hline $\mathrm{Pb}-\mathrm{I}-\mathrm{Pb}$ & $\mathrm{I}-\mathrm{Pb}$ & 1.536 \\
\hline $\mathrm{Pb}-\mathrm{I}-\mathrm{I}$ & $\mathrm{I}-\mathrm{I}$ & 1.535 \\
\hline $\mathrm{I}-\mathrm{I}-\mathrm{I}$ & $\mathrm{I}-\mathrm{Pb}$ & 1.535 \\
\hline $\mathrm{Pb}-\mathrm{I}-\mathrm{I}$ & $\mathrm{I}-\mathrm{Cs}$ & 1.533 \\
\hline $\mathrm{Cs}-\mathrm{I}-\mathrm{Pb}$ & $\mathrm{I}-\mathrm{Cs}$ & 1.532 \\
\hline $\mathrm{Cs}-\mathrm{I}-\mathrm{I}$ & $\mathrm{I}-\mathrm{Cs}$ & 1.531 \\
\hline $\mathrm{Cs}-\mathrm{I}-\mathrm{Pb}$ & $\mathrm{I}-\mathrm{Pb}$ & 1.529 \\
\hline $\mathrm{Cs}-\mathrm{I}-\mathrm{I}$ & $\mathrm{I}-\mathrm{I}$ & 1.529 \\
\hline $\mathrm{Pb}-\mathrm{I}-\mathrm{I}$ & $\mathrm{I}-\mathrm{Pb}$ & 1.526 \\
\hline $\mathrm{Cs}-\mathrm{I}-\mathrm{Pb}$ & $\mathrm{I}-\mathrm{I}$ & 1.523 \\
\hline
\end{tabular}




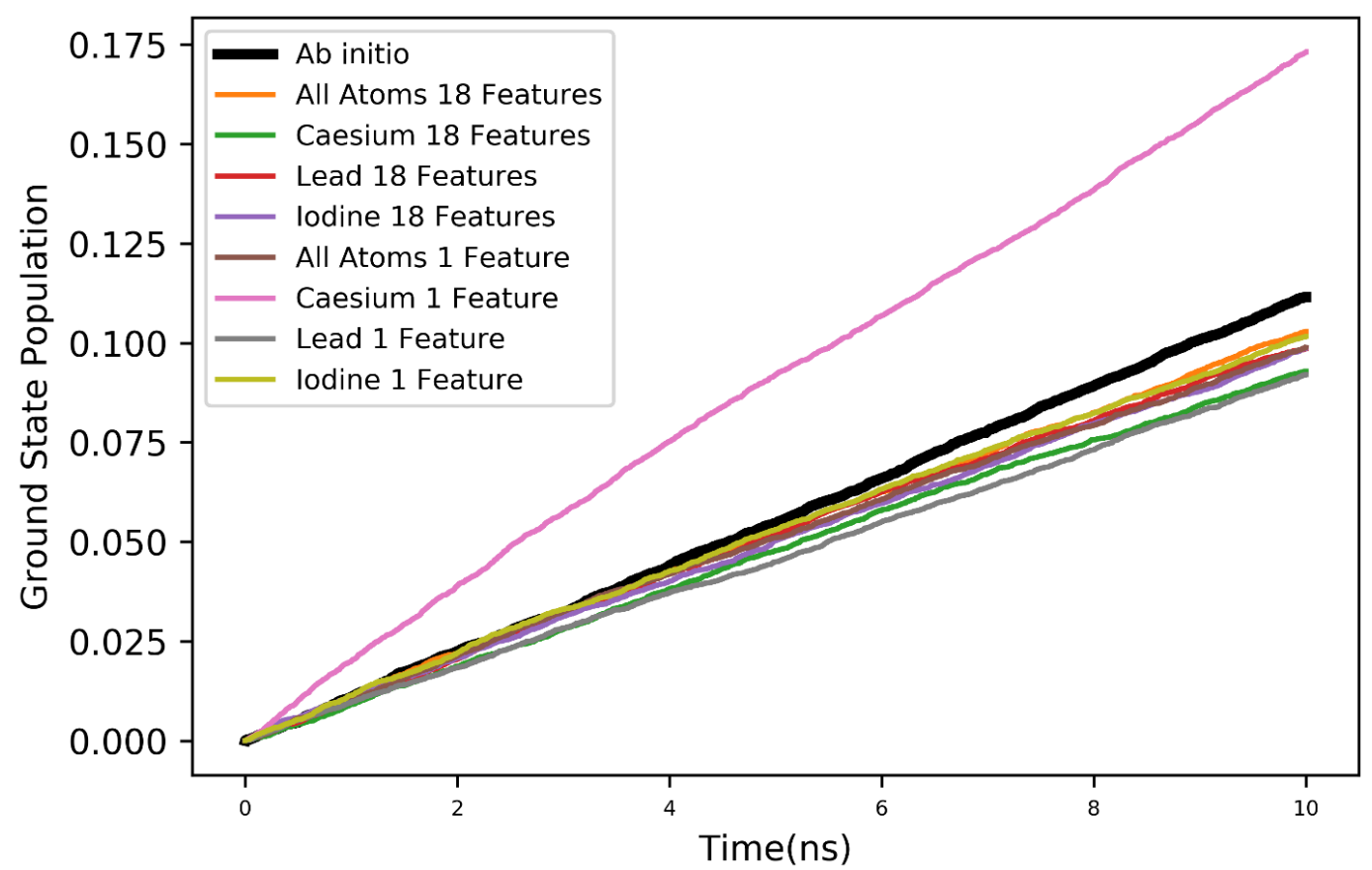

Figure S3: NA-MD results for pristine $\mathrm{CsPbI}_{3}$. Shown are the population of the ground state. The ab initio results are depicted via the bolded black line.

\section{References}

(1) Kresse, G.; Furthmüller, J. Efficient Iterative Schemes for Ab Initio Total-Energy Calculations Using a Plane-Wave Basis Set. Phys. Rev. B 1996, 54 (16), 11169-11186. https://doi.org/10.1103/PhysRevB.54.11169.

(2) Kresse, G.; Joubert, D. From Ultrasoft Pseudopotentials to the Projector Augmented-Wave Method. Phys. Rev. B 1999, 59 (3), 1758-1775. https://doi.org/10.1103/PhysRevB.59.1758.

(3) Chu, W.; Zheng, Q.; Akimov, A. V.; Zhao, J.; Saidi, W. A.; Prezhdo, O. V. Accurate Computation of Nonadiabatic Coupling with Projector Augmented-Wave Pseudopotentials. J. Phys. Chem. Lett. 2020, 11 (23), 10073-10080. https://doi.org/10.1021/acs.jpclett.0c03080.

(4) Chu, W.; Prezhdo, O. V. Concentric Approximation for Fast and Accurate Numerical Evaluation of Nonadiabatic Coupling with Projector Augmented-Wave Pseudopotentials. $J$. Phys. Chem. Lett. 2021, 12 (12), 3082-3089. https://doi.org/10.1021/acs.jpclett.0c03853.

(5) Jaeger, H. M.; Fischer, S.; Prezhdo, O. V. Decoherence-Induced Surface Hopping. J. Chem. Phys. 2012, 137 (22), 22A545. https://doi.org/10.1063/1.4757100.

(6) Akimov, A. V.; Prezhdo, O. V. Persistent Electronic Coherence Despite Rapid Loss of Electron-Nuclear Correlation. J. Phys. Chem. Lett. 2013, 4 (22), 3857-3864. https://doi.org/10.1021/jz402035z. 
(7) Habenicht, B. F.; Kamisaka, H.; Yamashita, K.; Prezhdo, O. V. Ab Initio Study of Vibrational Dephasing of Electronic Excitations in Semiconducting Carbon Nanotubes. Nano Lett. 2007, 7 (11), 3260-3265. https://doi.org/10.1021/n10710699.

(8) Akimov, A. V.; Prezhdo, O. V. The PYXAID Program for Non-Adiabatic Molecular Dynamics in Condensed Matter Systems. J. Chem. Theory Comput. 2013, 9 (11), 49594972. https://doi.org/10.1021/ct400641n.

(9) Akimov, A. V.; Prezhdo, O. V. Advanced Capabilities of the PYXAID Program: Integration Schemes, Decoherence Effects, Multiexcitonic States, and Field-Matter Interaction. J. Chem. Theory Comput. 2014, 10 (2), 789-804.

https://doi.org/10.1021/ct400934c. 